\title{
The Impact of Referral to Mental Health Services on Suicide Death Risk in Adolescent Suicide Survivors
}

\author{
Joonbeom Kim ${ }^{1,2}$, Sung Hee Hong ${ }^{1}$, and Hyun Ju Hong ${ }^{1,3}$ \\ ${ }^{1}$ Suicide and School Mental Health Institute, Anyang, Korea \\ ${ }^{2}$ Yonsei University, Interdisciplinary Graduate Program in Social Welfare Policy, Seoul, Korea \\ ${ }^{3}$ Department of Psychiatry, Hallym University Sacred Heart Hospital, Anyang, Korea
}

Objectives: This study aims to examine the effect of adolescent suicide survivors' experience on suicide death risk, and the effect of referral to mental health services (hereafter referral) in this regard.

Methods: This study used the data of 878 suicide-deceased and suicide-attempted adolescents aged 8-19 years, managed by the Suicide and School Mental Health Institute from 2016 to 2018.

Results: Regression analysis for main effects showed that although suicide experience had no direct effect on suicide death, non-referral status was associated with a greater risk of death by suicide. While the "non-suicide survivor with non-referral" and "suicide survivor with non-referral" groups showed 1.87 [adjusted odds ratio=1.87, 95\% confidence interval $(\mathrm{CI})=1.21-2.89$ ] and 4.59 (adjusted odds ratio= $4.59,95 \% \mathrm{CI}=2.02-10.42$ ) times higher odds of suicide death, respectively, the "suicide survivor with referral" group showed no difference compared to the "non-suicide survivor with referral" group.

Conclusion: From these findings, there is a need to strengthen referral to mental health services and apply complicated grief treatment to improve the mental health of adolescent suicide survivors.

Key Words: Suicide, Attempted; Suicide, Completed; Bereavement; Referral and consultation; Adolescent.

Received: July 6, 2020 / Revision: August 4, 2020 / Accepted: August 12, 2020

Address for correspondence: Hyun Ju Hong, Department of Psychiatry, Hallym University Sacred Heart Hospital, 22 Gwanpyeong-ro 170 beon-gil, Dongan-gu, Anyang 14068, Korea

Tel: +82-31-380-3750, Fax: +82-31-380-4118, E-mail: honghj88@gmail.com

\section{INTRODUCTION}

Since 2010, there have been over 13000 annual deaths by suicide in South Korea [1]. In this context, it is important to also attend to "suicide survivors"- people who had close social relationships with the suicide victim. Globally, the concept of suicide survivors is widely used to refer to people who experienced the loss of someone close due to suicide - those who have been involuntarily exposed to suicide through the death of a relative, friend, colleague, or neighbor $[2,3]$. In a broad sense, suicide survivors include all individuals exposed to the suicide of another, but the narrower meaning is usually restricted to close relationships such as family, friends, and neighbors [4]. Various studies roughly estimated that every suicide affects six or more suicide survivors on average [5]; however, this was refuted by a recent study [6] showing that every one of these deaths affects 135 suicide survivors. When this number is applied to the 13670 suicide victims

This is an Open Access article distributed under the terms of the Creative Commons Attribution Non-Commercial License (https://creativecommons.org/licenses/by-nc/4.0) which permits unrestricted non-commercial use, distribution, and reproduction in any medium, provided the original work is properly cited. in South Korea in 2018 [1], it gives over 1.8 million suicide survivors.

Suicide survivors frequently experience mental and psychosocial difficulties, and among them, children and adolescents especially are at risk of copycat suicides $[7,8]$. This is because the negative emotions associated with grief have an almost contagious effect of increasing suicidality [9]. In support of this, the "contagion hypothesis" [10] emphasizes that compared to adults, adolescents have less experience of mourning the death of someone they know, either due to suicide or other causes. Consequently, the experience of childhood suicide survivors is not limited simply to grief, but also has a negative psychological, emotional, and psychiatric ripple effect, which plays a key role in increasing the risk of suicide. In this regard, child survivors of suicide may experience a higher degree of shame, responsibility, and guilt compared to bereavement due to natural death or an accident $[11,12]$; when the victim is a family member, this leads to depression, guilt, post-traumatic stress disorder (PTSD), suicide ideation [13], and suicide attempts [14,15], even 3 years after the death. In an empirical study [16], the morbid- 
ity of psychiatric illness following a parent's death was analyzed in 4311096 children and adolescents aged 0-25 years using Swedish national data. The results showed that adolescent suicide survivors showed a 1.9-times higher risk ratio of major depressive disorder and a 1.7-fold higher risk ratio of suicide attempts compared to the rest of the sample (e.g., parents survived, parent died in accident). In particular, when the sample was divided by age groups, the risk ratio was 1.6 times higher in 13-17-year-olds, 1.7 times higher in 18-25-year-olds, and as much as 1.9 times higher in 0-12-year-olds. Childhood suicide survivors who lost a friend to suicide also showed persistent symptoms of depression, suicide ideation, and PTSD up to 8 months after death, and $27.3 \%$ of these individuals were reported to be in the high depression risk group [17]. These results show the need to investigate the effect of childhood suicide survivors' experiences on the risk of death by suicide.

A large number of studies have suggested that referral to specialized mental health institutions through schools can be a powerful protective factor that promotes mental health and reduces the risk of suicide $[18,19]$. This is because these programs reduce perceived barriers such as the stigma accompanying mental health treatment and encourage helpseeking behaviors [20], which are the first steps of the treatment [21]. Students with mental health problems are identified through regular mental health screening tests or observation by teachers or peers. Through this, it is possible to effectively refer them to specialized mental health institutions [22]. South Korea has implemented a universal screening system using an annual school-based mental health screening test, the Adolescent Personality and Mental Health Screening Questionnaire (APMHSQ) for 1st and 4th graders of elementary school, 1st graders of middle school and 1st graders of high school in the whole country. As a result, individuals identified as high-risk are referred to specialized mental health institutions outside schools, with a referral rate reaching $81.2 \%$ $(n=13754)$ in 2017 [23]. One previous study reported that the risk of suicide is lowered when adolescents with suicide attempts or self-injury are provided opportunities to consult with specialized institutions through school-based mental health services [24]. Given that suicide survivors can also be classified into the high mental health risk group, we hypothesized that providing opportunities to refer to hospitals or other specialized mental health institutions would further reduce the risk of death by suicide in this population. Therefore, in this study, we aimed to investigate the effect of referral to a specialized mental health institution on the relationship between risk of death by suicide and the experience of adolescent suicide survivors.

\section{METHOD}

\section{Participants}

This study used data from reports of student suicide attempts and deaths collected from the Korean Ministry of Education between 2016 and 2018. Currently, the Ministry of Education requires the regional offices of education to complete a report form for all suicide attempts and suicide deaths identified in schools. Thereafter, each regional office of education reports their collected data to the Ministry of Education, and the data is managed by an affiliated research center, the Suicide and School Mental Health Institute. There were 1597 cases reported between 2016 and 2018 (366 suicide deaths, 1231 suicide attempts); after excluding 719 cases for missing variables of interest (130 suicide deaths, 589 suicide attempts), the remaining 878 cases (236 suicide deaths, 642 suicide attempts) were included in the present analysis. This study was approved by the Hallym University Sacred Heart Hospital institutional review board (IRB No: 2016-I044).

\section{Measures}

\section{Suicide survivors}

Suicide survivors were defined using the narrow definition. We queried whether the student had experienced the suicide death of someone in their surrounding environment at the time of receipt of the incident report. Participants were coded as "0" (non-suicide survivor) and "1" (suicide survivor), where suicide survivors were those who had experienced the suicide death of a parent, brother, sister, relative, or friend.

\section{Death by suicide}

Death by suicide was based on the collected incident reports. Suicide attempts were coded as "0" and suicide deaths as "1."

\section{Referral to mental health services}

Referral to a specialized mental health institution was determined using a question in the "mental health support approaches" section, asking about details of referral to mental health services before the incident. Referral to mental health services was defined as visiting a specialized mental health institution outside of school, after 1) identification as the highrisk group based on APMHSQ, or 2) detecting mental health problems by observation of teachers, friends, or parents. Responses of "Not sure" were excluded; non-referral was coded as " 0 " and referral was coded as "1."

\section{Control variables}

As control variables, we used the sociodemographic fac- 
tors including sex, age, family economic status, and family structure. Sex and family structure were coded with dummy variables $(0=$ male, $1=$ female; $0=$ two-parent family, $1=$ singleparent/parentless family), while age and family economic status were used as continuous variables. We also controlled for means of suicide as a variable that could affect death by suicide. Based on a previous study [25], the means of suicide were divided into high lethality methods (e.g., hanging, jumping) and low lethality methods (e.g., drug poisoning, selfwounding), and these were coded as " 0 " (low lethality method) and "1" (high lethality method).

\section{Statistical analyses}

Frequency analysis was performed to characterize the variables of interest, and cross-tabulation analysis was conducted to test differences between suicide survivors and nonsuicide survivors. Correlation analysis was performed to analyze the correlations between the variables of being a suicide survivor, referral to mental health services, and death by suicide. To test the effects of being a suicide survivor and referral to mental health services on the risk of suicide death, we used logistic regression models. For the logistic regression analysis, the theoretical minimum risk group ("non-suicide survivor, referral") was used as the reference group, and the other three groups ("suicide survivor, non-referral;" "suicide survivor, referral;" "non-suicide survivor, non-referral") were dummified, and the odds ratios for suicide death were calculated. SPSS 25.0 (IBM Corp., Armonk, NY, USA) and STATA 16.0 (StataCorp Lp, College Station, TX, USA) were used for all statistical analyses.

\section{RESULTS}

\section{Characteristics of participants and comparison of variables according to suicide survivors}

Table 1 shows the sociodemographic characteristics of the participants, and the differences in variables of interest between suicide and non-suicide survivors. Of the 878 total participants, 109 persons (12.4\%) were suicide survivors. The most common relationship with the suicide victim was friend (38.5\%, $\mathrm{n}=42)$, followed by parent $(31.2 \%, \mathrm{n}=34)$, relative $(26.6 \%, n=29)$, and sibling $(3.7 \%, n=4)$. In terms of sociodemographic characteristics, there were far more females $(68.9 \%$, $\mathrm{n}=605)$ than males $(31.1 \%, \mathrm{n}=273)$. The most common educational level was high school $(50.8 \%, \mathrm{n}=446)$, the most common family structure was a two-parent family $(56.0 \%, \mathrm{n}=492)$, and the most common grade level was poor $(51.8 \%, \mathrm{n}=455)$, but there were no statistically significant differences in these variables between suicide survivors and non-suicide survivors. Meanwhile, economic status was significantly higher in suicide survivors $\left(\chi^{2}=6.357, \mathrm{p}<0.05\right)$, but no significant differences were found in the use of lethal methods or referral to mental health services. The percentage of suicide deaths was higher among suicide survivors $(37.6 \%, \mathrm{n}=41)$ than non-suicide survivors $(25.4 \%, \mathrm{n}=195)$, and this was statistically significant $\left(\chi^{2}=7.297, \mathrm{p}<0.01\right)$.

\section{Bivariate correlation of variables of interest}

Table 2 shows the results of correlation analysis for the variables of interest. Because the variables of interest were all categorical, the phi correlation was calculated. Suicide survivor status did not significantly correlate with referral to mental health services but showed a positive correlation with suicide death $(\mathrm{r}=0.119, \mathrm{p}<0.001)$. Referral to mental health services also showed a positive correlation with suicide death $(\mathrm{r}=0.184, \mathrm{p}<0.001)$.

\section{Model analysis}

Table 3 shows the results of analyzing the research models. Model 1 tested the primary effects of being a suicide survivor and referral to mental health services on death by suicide. First, suicide survivor status alone did not have a significant effect on suicide death, but not being referred to mental health services was associated with 2.08 times higher risk of death by suicide [adjusted odds ratio $(\mathrm{AOR})=2.08$, $\mathrm{p}<0.001]$. Among the control variables, female sex was associated with lower risk of suicide death $(\mathrm{AOR}=0.62, \mathrm{p}<0.05)$, whereas the risk of death by suicide increased with a higher educational level $(\mathrm{AOR}=2.49, \mathrm{p}<0.001)$ and with the use of lethal methods (e.g., jumping or hanging; $\mathrm{AOR}=46.54$, $\mathrm{p}<0.001)$. Model 2 tested how the risk of death by suicide was affected by the interaction between being a suicide survivor and referral to mental health services. Using the theoretical minimum risk group (non-suicide survivor and referral) as the reference, the risk of suicide death was 1.87 times higher in the non-suicide survivor and non-referral group ( $A O R=1.87, p<0.01)$. On the other hand, there was no significant effect on the suicide survivor and referral group. In other words, even if the participant was a suicide survivor, when they were referred to a mental health service, there was no significant difference in the risk of death by suicide compared to the group with the lowest risk. Meanwhile, the suicide survivor and non-referral group showed a 4.59 times higher risk of suicide death $(\mathrm{AOR}=4.59, \mathrm{p}<0.001)$. These results are displayed in Fig. 1.

\section{DISCUSSION}

Our findings demonstrate that adolescent suicide survivors have a higher risk of death by suicide and suggest that 
Table 1. Characteristics of participants and comparison of variables according to suicide survivors

\begin{tabular}{|c|c|c|c|c|}
\hline & \multirow{2}{*}{ Total $(n=878)$} & \multicolumn{2}{|c|}{ Suicide survivors } & \multirow{2}{*}{$\chi^{2}$} \\
\hline & & No $(n=769)$ & Yes $(n=109)$ & \\
\hline Gender & & & & 3.045 \\
\hline Male & $273(31.1)$ & $247(32.1)$ & $26(23.9)$ & \\
\hline Female & 605 (68.9) & $522(67.9)$ & $83(76.1)$ & \\
\hline School level & & & & 5.483 \\
\hline Elementary & $49(5.6)$ & $46(6.0)$ & $3(2.8)$ & \\
\hline Middle & $383(43.6)$ & $343(44.6)$ & $40(36.6)$ & \\
\hline High & $446(50.8)$ & $380(49.4)$ & $66(60.6)$ & \\
\hline Socioeconomic status & & & & $6.357^{*}$ \\
\hline High & $45(5.1)$ & $34(4.4)$ & $11(10.1)$ & \\
\hline Middle & $442(50.4)$ & $391(50.9)$ & $51(46.8)$ & \\
\hline Low & $391(44.5)$ & $344(44.7)$ & $47(43.1)$ & \\
\hline Family status & & & & 2.131 \\
\hline Parents & $492(56.0)$ & $438(57.0)$ & $54(49.5)$ & \\
\hline Others & $386(44.0)$ & $331(43.0)$ & $55(50.5)$ & \\
\hline Academic achievement & & & & 0.666 \\
\hline High & $126(14.4)$ & $108(14.0)$ & $18(16.5)$ & \\
\hline Middle & $297(33.8)$ & $263(34.2)$ & $34(31.2)$ & \\
\hline Low & $455(51.8)$ & $398(51.8)$ & $57(52.3)$ & \\
\hline Suicide means & & & & 1.205 \\
\hline Less-lethal & $470(53.5)$ & $417(54.2)$ & $53(48.6)$ & \\
\hline Lethal & $408(46.5)$ & $352(45.8)$ & $56(51.4)$ & \\
\hline Referral status & & & & 3.028 \\
\hline Referral & $399(45.4)$ & $341(44.3)$ & $58(53.2)$ & \\
\hline Non-referral & $479(54.6)$ & $428(55.7)$ & $51(46.8)$ & \\
\hline Suicide death & & & & $7.297^{\dagger}$ \\
\hline No & $642(73.1)$ & $574(74.6)$ & $68(62.4)$ & \\
\hline Yes & $236(26.9)$ & $195(25.4)$ & $41(37.6)$ & \\
\hline Relationships with suicide decedents ${ }^{\ddagger}$ & & & & - \\
\hline Parents & $34(31.2)$ & - & $34(31.2)$ & \\
\hline Siblings & $4(3.7)$ & - & $4(3.7)$ & \\
\hline Relatives & $29(26.6)$ & - & $29(26.6)$ & \\
\hline Friends & $42(38.5)$ & - & $42(38.5)$ & \\
\hline
\end{tabular}

Values are presented as $n(\%)$ unless otherwise indicated. ${ }^{*} p<0.05,{ }^{\dagger} p<0.01$, ${ }^{\ddagger}$ total $n=109$

referral to mental health services could mitigate that risk. The major findings are discussed below. First, 109 of the 878 total participants (12.4\%) were suicide survivors. This is consistent with a previous study showing that the rate of suicide survivors was over $11 \%$ [13]. Specifically, the most common relationship of the suicide victim to the suicide survivor was a friend $(38.5 \%, \mathrm{n}=42)$, followed by a parent $(31.2 \%, \mathrm{n}=34)$, a relative $(26.6 \%, \mathrm{n}=29)$, and a sibling $(3.7 \%, \mathrm{n}=4)$. If parents, siblings, and other relatives are all categorized as family, the percentage increases to $61.5 \%(\mathrm{n}=67)$, meaning that, for approximately 3 out of 5 suicide survivors, the suicide victim is a family member. However, when we analyzed the primary effects in our research model, being a suicide survivor alone
Table 2. Bivariate correlation among variables of interests

\begin{tabular}{lccc}
\hline & 1 & 2 & 3 \\
\hline 1. Suicide survivors & - & & \\
2. Referral status & 0.051 & - & \\
3. Suicide death & $0.119^{*}$ & $0.184^{*}$ & - \\
\hline${ }^{*} \mathrm{p}<0.001$ & & &
\end{tabular}

did not result in a statistically significant increase in the risk of death by suicide. In general, existing studies have reported that adolescent suicide survivors are vulnerable to develop internalizing disorders such as depression or anxiety [26], and are at higher risk of direct suicide attempts $[12,15]$. However, unlike these previous studies, our study sample only 
Table 3. The research model results

\begin{tabular}{|c|c|c|c|c|}
\hline & \multicolumn{2}{|c|}{ Model $1(n=878)$} & \multicolumn{2}{|c|}{ Model $2(n=878)$} \\
\hline & AOR & $95 \% \mathrm{Cl}$ & AOR & $95 \% \mathrm{Cl}$ \\
\hline \multicolumn{5}{|l|}{ Gender } \\
\hline Male (ref) & 1.00 & & 1.00 & \\
\hline Female & $0.62^{*}$ & $0.41-0.94$ & $0.62 *$ & $0.41-0.94$ \\
\hline School level & $2.49^{\ddagger}$ & $1.72-3.45$ & $2.48^{\ddagger}$ & $1.74-3.55$ \\
\hline Socioeconomic status & $0.56^{\dagger}$ & $0.38-0.84$ & $0.56^{\dagger}$ & $0.38-0.83$ \\
\hline \multicolumn{5}{|l|}{ Family status } \\
\hline Parents (ref) & 1.00 & & 1.00 & \\
\hline Others & 1.07 & $0.66-1.70$ & 1.07 & $0.67-1.71$ \\
\hline Academic achievement & 0.77 & $0.58-1.01$ & 0.77 & $0.58-1.02$ \\
\hline \multicolumn{5}{|l|}{ Suicide means } \\
\hline Less-lethal (ref) & 1.00 & & 1.00 & \\
\hline Lethal & $46.54^{\ddagger}$ & $23.34-90.43$ & $46.55^{\ddagger}$ & $23.56-91.96$ \\
\hline \multicolumn{5}{|l|}{ SS } \\
\hline No (ref) & 1.00 & & & \\
\hline Yes & 1.71 & $0.92-3.15$ & & \\
\hline \multicolumn{5}{|l|}{ Referral status } \\
\hline Referral (ref) & 1.00 & & & \\
\hline Non-referral & $2.08^{\ddagger}$ & $1.38-3.12$ & & \\
\hline \multicolumn{5}{|l|}{ Groups } \\
\hline Non-SS and Referral (ref) & & & 1.00 & $1.21-2.89$ \\
\hline Non-SS and Non-referral & & & $1.87^{\dagger}$ & $0.39-2.70$ \\
\hline SS and Referral & & & 1.03 & $2.02-10.42$ \\
\hline SS and Non-referral & & & $4.59^{\ddagger}$ & \\
\hline Constant & $0.02^{\ddagger}$ & $0.00-0.12$ & $0.02^{\ddagger}$ & $0.00-0.06$ \\
\hline $\operatorname{LR~chi}^{2}(\mathrm{n})$ & \multicolumn{2}{|c|}{$437.61(8)^{\ddagger}$} & \multicolumn{2}{|c|}{$439.47(9)^{\ddagger}$} \\
\hline Pseudo $\mathrm{R}^{2}$ & \multicolumn{2}{|c|}{0.428} & \multicolumn{2}{|c|}{0.430} \\
\hline
\end{tabular}

included the high-risk group consisting of suicide attempters and completers, and this could be surmised to be the reason for the discrepancy. On the other hand, not being referred to a mental health service was associated with a 2.08-time higher risk of death by suicide $(\mathrm{AOR}=2.08, \mathrm{p}<0.001)$. Our novel finding is that referral of the high mental health risk groups to mental health services can reduce the risk of death by suicide. Moreover, when we analyzed the interaction between non-referral and suicide survivor status, the risk of suicide death increased by 4.59 ; this indicates the importance of identifying vulnerable individuals, such as suicide survivors, and providing referral to mental health services.

These results demonstrate the need to give attention to adolescent suicide survivors. Due to the nature of adolescents as a group, their intimate social network mostly consists of home and school, and these environments are both closely associated with adolescent development. One empirical study [14] sampled 19376 university students and extracted only those with death by suicide in the family; the authors then analyzed the effects of a family history of suicide on suicide attempts, and reported that even when controlling for psychiatric symptoms (The General Health Questionnaire-12, GHQ-12), the risk of suicide attempts was around 3 times higher in students with a family history of suicide. On the other hand, a study on the exposure to the death of a friend by suicide [27], administered the Child Report of Post-traumatic Symptoms (CROPS) test to 956 adolescents at schools with suicide deaths, and found that only $8.6 \%$ of the subjects were classified as high-risk suicide survivors. These results demonstrate that the experiences of suicide survivors are closely related to the intimacy and quality of the relationship with the victim [28], and that, in addition to direct intervention for family suicide survivors, it is also of the utmost importance to screen for the high-risk group in the case of friend relationships.

Key considerations for mental health services for suicide survivors can be divided into interventions for complicated grief through specialized mental health services including 


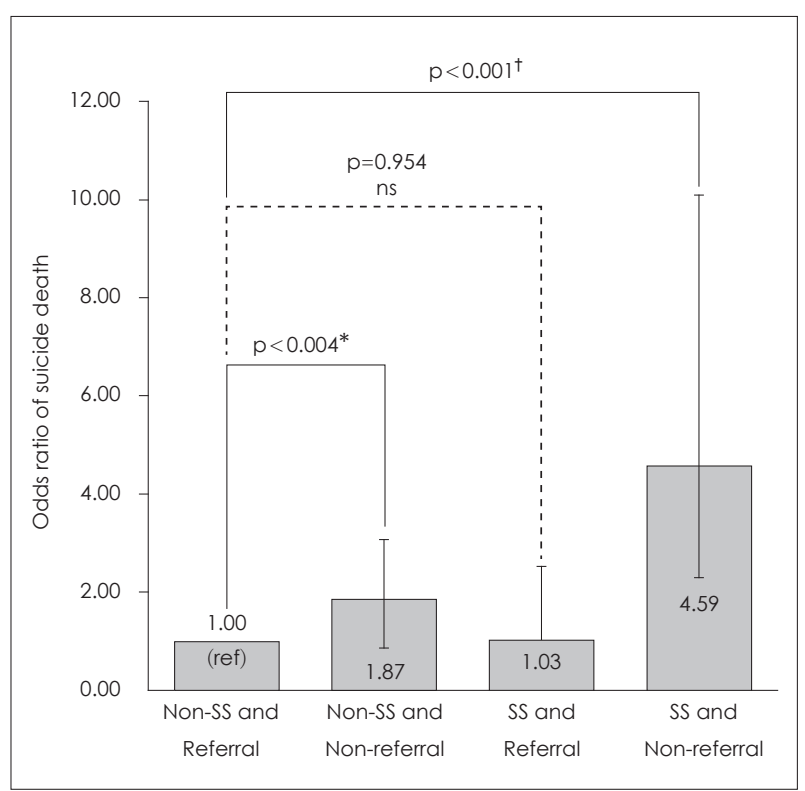

Fig. 1. Results for the group differences to suicide death risk. ${ }^{*} \mathrm{p}<0.01$, $t p<0.001$. ns: not significant, ref: reference, Ss: suicide survivors.

psychiatric treatment and for alleviating the stigma associated with screening. First, in a study performing psychological autopsy on adolescent suicide survivors [29], up to $95 \%$ of subjects showed psychiatric difficulties meeting clinical criteria. Consequently, when providing mental health services for the high-risk group, providing psychiatric treatment may be essential. Meanwhile, when providing treatment for suicide survivors, it is crucial to deal with complicated grief. Complicated grief is common in adolescent suicide survivors who have experienced bereavement due to suicide in an intimate relationship, and it is reported to be closely associated with psychiatric symptoms such as depression [30], anxiety, PTSD, and suicide ideation [31]. Some studies have suggested that there may be a long time-gap between the time of bereavement and the expression of psychiatric symptoms such as depression and anxiety [31], and there is a need to prioritize treatment of complicated grief [32]. This is derived from the fact that complicated grief is accompanied by difficulty accepting bereavement, anger about bereavement, and loss of meaning in life after bereavement, and can persist throughout a lifetime, existing as a clearly separate but related construct with other psychiatric symptoms such as depression, anxiety, and PTSD [32,33]. One previous study [34] has demonstrated that the core resources to alleviate the symptoms of grief in adolescents are hope and positive expectations about the future. Therefore, in suicide survivors, it is important to prioritize treatment of complicated grief by improving the ability to cope with grief-inducing incidents, correcting cognitive distortions, and reinforcing the positivity of life via supportive resources in the patient's surrounding environment.
Next, environmental interventions need to be implemented simultaneously to alleviate the stigma experienced by suicide survivors. One study [12] demonstrated that perceived stigma accompanying the experience of suicide survivors was a key factor influencing suicide attempts and emphasized the importance of eliminating perceived stigma as a core element of interventions in suicide survivors. This indicates that the experiences of suicide survivors bring shock and grief, while also increasing anxiety, depression, and suicidality; as a result, survivors are concerned about the stigma of being classified into the high mental health risk group. Based on these results, interventions for adolescent suicide survivors should focus on creating a supportive environment that can minimize the stigma associated with screening. Indeed, one study [27] introduced the strategy of forming a supportive environmental system to alleviate stigma and applied this school-based crisis intervention to 956 adolescents in schools who had experienced suicide deaths. Education, including mental health knowledge and proper understanding of grief, was provided to all members of the school, while specialized mental health treatment and supportive counseling services were provided to high-risk students. In follow-up testing 5 months after the interventions, the high-risk group had decreased by around $66.2 \%$. Therefore, in addition to personal medical treatment, linkage with comprehensive mental health services, including environmental resources, is expected to reduce the risk of death by suicide among suicide survivors.

This study had several limitations. First, the data we used is from completed or attempted suicide reports collected by the Ministry of Education, and so it is possible that we may have missed unreported incidents, or that incidents may have been downscaled in the reports. In addition, suicide attempts can be divided more specifically into categories, such as aborted or self-interrupted attempts, as shown in the ColombiaSuicide Severity Rating Scale, but this was not possible with the data used in this study. In future studies, suicide attempts will need to be classified more precisely, and this will require face-to-face assessment methods, such as caregiver interviews, when collecting completed or attempted suicide incident reports. Also, because this study uses cross-sectional data, there are limitations in generalizing the causative relationships between the variables of interest. Second, the data in this study are based on completed and attempted suicide incident reports submitted by schools to the regional offices of education after the incident. However, because the respondents in each report are different (e.g., homeroom teacher, school counselor, school principal), the responses may differ in accuracy. Third, "referral to mental health services" included all instances of specialist institutions outside of school, regardless of the type of institution. It will be necessary to dif- 
ferentiate the effects of the type of institution providing mental health services on the risk of death by suicide. Fourth, there was a large loss of data because about half of the original data $(\mathrm{n}=719)$ were lacking the variable-of-interest of being a suicide survivor. As a result, we were unable to include other variables that could affect suicide death (e.g., previous suicide attempts) in the research model, because they resulted in further missing values. These will need to be considered in further studies.

\section{CONCLUSION}

This study analyzed how the risk of death by suicide was affected by being an adolescent suicide survivor who had lost someone close to suicide, and by referral to a specialized mental health institution. Four groups were defined based on whether the participant was a suicide survivor and whether they were referred to a mental health service; compared to the reference group of "non-suicide survivor, referral", the "non-suicide survivor, non-referral" group showed a 1.87time higher risk of death by suicide, and the "suicide survivor, non-referral" group showed a 4.59-time higher risk of death by suicide. On the other hand, the "suicide survivor, referral" group did not showed significant difference with the reference group.

\section{Acknowledgments}

This work was supported by the Ministry of Education of the Republic of Korea and the National Research Foundation of Korea (NRF-2018S1A5B8A02081988).

\section{Conflicts of Interest}

The authors have no potential conflicts of interest to disclose.

\section{Author Contributions}

Conceptualization: Joonbeom Kim. Data curation: all authors. Formal analysis: Joonbeom Kim. Funding acquisition: Hyun Ju Hong. Investigation: all authors. Methodology: Joonbeom Kim. Project administration: Hyun Ju Hong, Sung Hee Hong. Resources: Hyun Ju Hong. Software: Joonbeom Kim. Supervision: Hyun Ju Hong, Sung Hee Hong. Validation: Hyun Ju Hong. Visualization: Joonbeom Kim, Sung Hee Hong. Writing_original draft: Joonbeom Kim. Writing_-review \& editing: Hyun Ju Hong, Sung Hee Hong.

\section{ORCID iDs}

Joonbeom Kim https://orcid.org/0000-0002-1487-5882

Sung Hee Hong https://orcid.org/0000-0003-4347-6976

Hyun Ju Hong https://orcid.org/0000-0002-6348-9996

\section{REFERENCES}

1) Korea Suicide Prevention Center. 2020 Suicide Prevention White Paper. 1st ed. Seoul: Korea Suicide Prevention Center;2020. p.20.

2) Cerel J, Maple M, Aldrich R, van de Venne J. Exposure to suicide and identification as survivor. Results from a random-digit dial survey. Crisis 2013;34:413-419.
3) Song IH, Kwon SW, Kim JE. Association between suicidal ideation and exposure to suicide in social relationships among family, friend, and acquaintance survivors in South Korea. Suicide Life Threat Behav 2015;45:376-390.

4) Knieper AJ. The suicide survivor's grief and recovery. Suicide Life Threat Behav 1999;29:353-364.

5) Shneidman EF. Introduction. In: Cain AC, editor. Survivors of suicide. Springfield: Thomas;1972. p.ix-xi.

6) Cerel J, Brown MM, Maple M, Singleton M, van de Venne J, Moore M, et al. How many people are exposed to suicide? Not six. Suicide Life Threat Behav 2019;49:529-534.

7) Gould MS, Lake AM. The contagion of suicidal behavior. Contagion of Violence: Workshop Summary; 2013. National Academies Press [cited 2020 June 21]. Available from URL: https://www. ncbi.nlm.nih.gov/books/NBK207262/.

8) Insel BJ, Gould MS. Impact of modeling on adolescent suicidal behavior. Psychiatr Clin North Am 2008;31:293-316.

9) Cheng Q, Li H, Silenzio V, Caine ED. Suicide contagion: a systematic review of definitions and research utility. PLoS One 2014;9: e108724.

10) Brent DA, Kerr MM, Goldstein C, Bozigar J, Wartella M, Allan MJ. An outbreak of suicide and suicidal behavior in a high school. J Am Acad Child Adolesc Psychiatry 1989;28:918-924.

11) Tal I, Mauro C, Reynolds CF 3rd, Shear MK, Simon N, Lebowitz B, et al. Complicated grief after suicide bereavement and other causes of death. Death Stud 2017;41:267-275.

12) Pitman AL, Osborn DP, Rantell K, King MB. Bereavement by suicide as a risk factor for suicide attempt: a cross-sectional national UK-wide study of 3432 young bereaved adults. BMJ Open 2016;6: e009948.

13) Song IH, Kwon SW, Kim JE. Suicide attempts by family members and peers and their impact on suicidal ideation among adolescents: an analysis of the mediating effect of depressive mood. Korean Journal of Social Welfare Research 2016;49:29-47.

14) Wang YG, Chen S, Xu ZM, Shen ZH, Wang YQ, He XY, et al. Family history of suicide and high motor impulsivity distinguish suicide attempters from suicide ideators among college students. J Psychiatr Res 2017;90:21-25.

15) Jakobsen IS, Christiansen E. Young people's risk of suicide attempts in relation to parental death: a population-based register study. $\mathrm{J}$ Child Psychol Psychiatry 2011;52:176-183.

16) Wilcox HC, Kuramoto SJ, Lichtenstein P, Långström N, Brent DA, Runeson B. Psychiatric morbidity, violent crime, and suicide among children and adolescents exposed to parental death. J Am Acad Child Adolesc Psychiatry 2010;49:514-523; quiz 530.

17) Kang NR, Chung US, Kwack YS. Impact of peer's suicide on mental health of adolescents. J Korean Acad Child Adolesc Psychiatry 2015;26:266-272.

18) Anderson JK, Ford T, Soneson E, Coon JT, Humphrey A, Rogers M, et al. A systematic review of effectiveness and cost-effectiveness of school-based identification of children and young people at risk of, or currently experiencing mental health difficulties. Psychol Med 2019;49:9-19.

19) Gaete J, Martinez V, Fritsch R, Rojas G, Montgomery AA, Araya R. Indicated school-based intervention to improve depressive symptoms among at risk Chilean adolescents: a randomized controlled trial. BMC Psychiatry 2016;16:276.

20) Klimes-Dougan B, Klingbeil DA, Meller SJ. The impact of universal suicide-prevention programs on the help-seeking attitudes and behaviors of youths. Crisis 2013;34:82-97.

21) Baiden P, Fallon B. Examining the association between suicidal behaviors and referral for mental health services among children involved in the child welfare system in Ontario, Canada. Child Abuse Negl 2018;79:115-124.

22) Gould MS, Marrocco FA, Hoagwood K, Kleinman M, Amakawa L, 
Altschuler E. Service use by at-risk youths after school-based suicide screening. J Am Acad Child Adolesc Psychiatry 2009;48:11931201.

23) Kim J, Hong S, Hong H. Exploring demands and directions of field experts on ministry of education's school-based suicide prevention policy. Journal of Education \& Culture 2019;25:5-31.

24) Husky MM, Kaplan A, McGuire L, Flynn L, Chrostowski C, Olfson $\mathrm{M}$. Identifying adolescents at risk through voluntary schoolbased mental health screening. J Adolesc 2011;34:505-511.

25) Cha JH, Ahn ME, Kim DW, Lee SK, Lee CH, Kim SM, et al. Clinical characteristics of intentional self-harm inpatient with lethal methods. J Korean Soc Emerg Med 2019;30:419-427.

26) Stikkelbroek Y, Bodden DH, Reitz E, Vollebergh WA, van Baar AL. Mental health of adolescents before and after the death of a parent or sibling. Eur Child Adolesc Psychiatry 2016;25:49-59.

27) Cha JM, Kim JE, Kim MA, Shim B, Cha MJ, Lee JJ, et al. Five months follow-up study of school-based crisis intervention for Korean high school students who experienced a peer suicide. J Korean Med Sci 2018;33:e192.

28) Mash HB, Fullerton CS, Shear MK, Ursano RJ. Complicated grief and depression in young adults: personality and relationship quality. J Nerv Ment Dis 2014;202:539-543.

29) O'Connor RC, Nock MK. The psychology of suicidal behaviour. Lancet Psychiatry 2014;1:73-85.

30) Dickens N. Prevalence of complicated grief and posttraumatic stress disorder in children and adolescents following sibling death. The Family Journal 2014;22:119-126.

31) Melhem NM, Moritz G, Walker M, Shear MK, Brent D. Phenomenology and correlates of complicated grief in children and adolescents. J Am Acad Child Adolesc Psychiatry 2007;46:493-499.

32) Salloum A, Bjoerke A, Johnco C. The associations of complicated grief, depression, posttraumatic growth, and hope among bereaved youth. Omega (Westport) 2019;79:157-173.

33) Brent DA, Melhem NM, Masten AS, Porta G, Payne MW. Longitudinal effects of parental bereavement on adolescent developmental competence. J Clin Child Adolesc Psychol 2012;41:778-791.

34) Kilmer RP, Gil-Rivas V, Griese B, Hardy SJ, Hafstad GS, Alisic E. Posttraumatic growth in children and youth: clinical implications of an emerging research literature. Am J Orthopsychiatry 2014;84: 506-518. 